\title{
$\begin{array}{r}\text { WAGENINGEN } \\ \hline \\ \hline\end{array}$
}

Dietary diversity affects feeding behaviour of suckling piglets

Middelkoop, A., Choudhury, R., Gerrits, W. J. J., Kemp, B., Kleerebezem, M., \& Bolhuis, J. E.

This is a "Post-Print" accepted manuscript, which has been published in "Applied Animal Behaviour Science"

This version is distributed under a non-commercial no derivatives Creative Commons (c) $(\mathcal{Q} \Theta \Theta(\mathrm{CC}-\mathrm{BY}-\mathrm{NC}-\mathrm{ND})$ user license, which permits use, distribution, and reproduction in any medium, provided the original work is properly cited and not used for commercial purposes. Further, the restriction applies that if you remix, transform, or build upon the material, you may not distribute the modified material.

Please cite this publication as follows:

Middelkoop, A., Choudhury, R., Gerrits, W. J. J., Kemp, B., Kleerebezem, M., \& Bolhuis, J. E. (2018). Dietary diversity affects feeding behaviour of suckling piglets. Applied Animal Behaviour Science, 205, 151-158. DOI:

10.1016/j.applanim.2018.05.006

You can download the published version at:

https://doi.org/10.1016/j.applanim.2018.05.006 


\section{Dietary diversity affects feeding behaviour of suckling piglets}

Anouschka Middelkoop ${ }^{1}$, Raka Choudhury ${ }^{2}$, Walter J.J. Gerrits ${ }^{3}$, Bas Kemp ${ }^{1}$, Michiel Kleerebezem², J. Elizabeth Bolhuis ${ }^{1}$

${ }^{1}$ Adaptation Physiology Group, Department of Animal Sciences, Wageningen University \& Research, P.O. Box 338, 6700 AH Wageningen, The Netherlands

${ }^{2}$ Host-Microbe Interactomics Group, Department of Animal Sciences, Wageningen University \& Research, P.O. Box 338, 6700 AH Wageningen, The Netherlands

${ }^{3}$ Animal Nutrition Group, Department of Animal Sciences, Wageningen University \& Research, P.O. Box 338, 6700 AH Wageningen, The Netherlands

Corresponding author: Anouschka Middelkoop.

Address: P.O. Box 338, 6700 AH Wageningen, The Netherlands

Email: anouschka.middelkoop@wur.nl

\section{Abstract}

Stimulating solid feed intake in suckling piglets is important to facilitate the weaning transition, exemplified by the positive correlation between pre- and post-weaning feed intake. The present study compared the effect of dietary diversity (i.e. offering two feeds simultaneously) and flavour novelty (i.e. regularly changing the flavour of one feed) on the feeding behaviour and performance of suckling piglets until weaning at day 22. It was hypothesized that varying multiple sensory properties of the feed, by presentation of the feed in a more diverse form, stimulates pre-weaning feed intake. Piglets received ad libitum feed from 2 days of age in two feeders per pen (choice feeding set-up). One group of piglets (dietary diversity (DD), $n=10$ litters) were given feed A and feed B which differed in production method, size, flavour, ingredient composition and nutrient profile, smell, texture and colour. The other group of piglets 
(flavour novelty ( $F N), n=9$ litters) received feed $A$ plus feed $A$ to which one of 4 flavours were added from day 6 in a daily sequential order. Feeding behaviour was studied by weighing feed remains $(d 6,12,16,22)$ and by live observations (4-min scan sampling, 6h/d; d9, 14, 21; n=6 litters per treatment). Observations were also used to discriminate 'eaters' from 'non-eaters'. All piglets were weighed at $\mathrm{d} 2,6$ and 22. Piglets did not prefer feed A (d2-22: $1.4 \pm 0.16 \mathrm{~kg} / \mathrm{litter})$ over B (1.6 \pm 0.18$)$ within DD nor had a preference for feed A with (d6-22: $1.1 \pm 0.06 \mathrm{~kg} / \mathrm{litter})$ or without additional flavours $(0.9 \pm 0.07)$ within FN. Nevertheless, DD-litters (d2-22: $3.0 \pm 0.32 \mathrm{~kg}$ ) ate significantly more than FN-litters $(2.0 \pm 0.12 \mathrm{~kg} ; P=0.02)$ and explored the feed 2.6 times more at $\mathrm{d} 14(P=0.001)$. Furthermore feed $\mathrm{A}$, the common feed provided in DD and FN, was more consumed in DD (d2-22: $1.4 \pm 0.16 \mathrm{~kg})$ compared to FN $(1.0 \pm 0.07 \mathrm{~kg} ; P=0.04)$. The percentage of eaters within a litter did not differ over time between DD (d9: 26\%, d14: 78\%, d21: $94 \%)$ and FN (20\%, 71\% and 97\%) and no effect was found on pre-weaning weight gain. In conclusion, this study showed that provision of dietary diversity to suckling piglets stimulated their feed exploration and intake more than dietary flavour novelty only, but did not enhance the percentage of piglets within a litter that consume the feed or their growth performance. These data suggest that dietary diversity could be an innovative feeding strategy to stimulate solid feed intake in suckling piglets.

Key-words: Behaviour; Creep feed; Dietary diversity; Feed intake; Flavour; Piglet.

\section{Highlights}

- We studied an innovative feeding strategy to increase solid feed intake pre-weaning

- Dietary diversity stimulated feed intake of suckling piglets more than flavour novelty

- The percentage of eaters was not affected, meaning a higher feed intake per piglet

- Our results support that the more diverse the feeds are, the greater their intake

- Intrinsic exploration and sensory-specific satiety may underlie this 


\section{Implications}

This study indicates that provision of diverse solid feed types (i.e. varying in multiple sensory properties) before weaning can enhance feed exploration and intake by suckling piglets compared to solid feed types that vary in flavour only. Piglets with a high uptake of solid feed before weaning have been shown to outperform piglets with a low pre-weaning uptake of solid feed initially after weaning in terms of feed intake and growth performance (Carstensen et al., 2005; Pluske et al., 2007). As such, pre-weaning dietary diversity may benefit postweaning piglet (gut) health, welfare and performance.

\section{Introduction}

In conventional pig farming, piglets are removed from the sow at 3 to 4 weeks of age. Piglets weaned early and abruptly are challenged with numerous concurrent stress factors such as changes in social structure, environment and diet. The latter includes deprivation of sow's milk and a change to a weaner diet, which usually consists of solid feed. Weaning-related stress is associated with a delayed and low feed intake in the initial post-weaning period (Bruininx et al., 2002 and 2004). The combination of stress, acute fasting, shift in diet physical form and subsequent introduction of novel food antigens at weaning results in undesirable changes in gut morphology and microbiota, thereby increasing the risk for maldigestion and absorption, enteric pathogen colonization, post-weaning diarrhoea and growth stasis, as reviewed by Heo et al. (2013). The physical form and composition of the post-weaning diet also play a crucial role on gut health for newly weaned pigs (Sander et al., 2012; Torrallardona et al., 2012).

Creep feed is an optional provision for suckling piglets to familiarise them with solid feed prior to weaning. There is evidence that the consumption of solid feed during lactation has a positive effect on solid feed intake in the initial post-weaning period and growth performance of piglets around weaning (Bruininx et al., 2002 and 2004; Kuller et al., 2007; 
Sulabo et al., 2010). Moreover, these effects are especially pronounced in piglets with an early uptake of creep feed (Klindt, 2003; Van den Brand et al., 2014) and/or a high creep feed consumption level (Bruininx et al., 2004; Carstensen et al., 2005; Pluske et al., 2007). The latter is supported by the highly positive correlation between feed intake pre-weaning and feed intake and growth initially after weaning (Berkeveld et al., 2007; Kuller et al., 2004). However, such studies have also shown that the creep feeding behaviour of conventional suckling piglets is still immature: first, a significant and highly variable proportion of piglets starts to consume creep feed only relatively shortly before weaning or fails to consume any creep feed until weaning (e.g. Pluske et al., 2007; Tucker et al., 2010; Van der Meulen et al., 2010); and second, creep feed consumption by suckling piglets is low, unpredictable and variable between and within litters (Bruininx et al., 2002 and 2004; Carstensen et al., 2005; Pajor et al., 1991). It is therefore important to find strategies that initiate early creep feed intake, stimulate the number of piglets consuming the creep feed, and enhance its consumption level to create more robust piglets around weaning.

In a (semi-)natural environment, the development of feeding behaviour already starts on the first days of a piglet's life by digging soft soil and exploration of feed and non-feed substrates by rooting, nosing, chewing and biting (Gundlach, 1986; Petersen, 1994). Pigs are opportunistic and omnivorous feeders and known to consume an extensive variety of food items, ranging from plant material, like nuts, roots, seeds, tubers and products of animal origin like earthworms (Hanson and Karstad, 1959; Pinna et al., 2007). Suckling piglets thereby encounter a variety of (novel) food items under (semi-)natural conditions and have been observed sampling leaves, mushrooms, acorns and corn (Gundlach, 1986; Meynhardt, 1980; Petersen, 1994). In contrast, conventional suckling piglets are mostly offered a single diet. We hypothesize that presentation of the creep feed in a more diverse and/or novel form stimulates their exploratory and feeding behaviour.

Dietary variety consists of feeds that differ in at least one sensory property (Raynor and Epstein, 2001), of which flavour is mostly studied. Dietary variety, either simultaneous or 
successive, has been shown to alter feeding behaviour and increase feed intake in humans (Rolls et al., 1981), rats (Treit et al., 1983; Rolls et al., 1983) and sheep (Distel et al., 2007; Villalba et al., 2011). Similar effects have been found recently in suckling piglets as well (Adeleye et al., 2014). These studies indicate that varying one sensory property of the feed (e.g. flavour) can already have a stimulatory effect on feed intake. It is hypothesized however, that the more diverse the feeds are, the more rewarding it is to switch between them and to consume more in total (Rolls et al., 1981). Our study thus aimed to compare the effect of dietary diversity (i.e. offering two feeds simultaneously) and flavour novelty (i.e. regularly changing the flavour of one feed) on the feeding behaviour and performance of suckling piglets.

\section{Materials and methods}

\subsection{Animals and housing}

The Animal Care and Use committee of Wageningen University \& Research (Wageningen, The Netherlands) approved the protocol of the experiment. Top Pi $x$ Topigs-20 piglets (both sexes) from 19 multiparous sows (range parity: 1 to 7 ) were used in a two-choice feeding set-up. About one week before farrowing, the sows were moved to two adjacent farrowing rooms and were housed in individual conventional pens $(2.2 \times 2.0 \mathrm{~m})$ without bedding material. The pen was equipped with a farrowing crate including feed trough, drinking nipple and a metal chain with ball for the sows (not accessible to the piglets) and a drinking nipple for the piglets. Sows were fed a commercially available diet twice a day. The pen consisted of $80 \%$ slatted floor and $20 \%$ solid floor, with an infrared lamp above it, as a piglet nest area. At one day of age piglets were weighed, received an ear tag, received an intramuscular iron injection of $1 \mathrm{cc}$, and were tail docked and teeth clipped. Within 2 days after birth, litter size was standardized to 13-15 piglets per litter by cross-fostering. At 5 days of age, male piglets were castrated. Piglets were vaccinated against Mycoplasma, Circo and E. coli at 6 
days before weaning at $22.3 \pm 0.05$ days of age. Room temperature was $25^{\circ} \mathrm{C}$ around farrowing and was gradually decreased to $22{ }^{\circ} \mathrm{C}$ until weaning. Artificial lighting was provided between 07:00 and 18:00 $\mathrm{h}$.

\subsection{Dietary treatment}

Piglets received feed ad libitum from 2 days of age in two concrete round creep feed bowls (diameter of $21 \mathrm{~cm}$ ), each having four feeding places, per pen. The amount of creep feed in the feed bowls was checked at least twice daily to prevent the bowls of getting empty. To minimize spillage of creep feed, the bowls had partitions and were attached to the solid floor of the pen, each positioned at one side of the piglet nest area. The position of the bowls was switched on a daily basis within litters to ensure that feed intake was not affected by feeder position preference.

One group of piglets (dietary diversity (DD), $n=10$ litters) were given feed $A$ (experimental diet, Animal Nutrition Group, Wageningen University \& Research, Wageningen, The Netherlands) and feed B (commercial diet, Baby Big XL, Coppens Diervoeding, Helmond, the Netherlands) which differed in production method, size, flavour, ingredient composition and nutrient profile, smell, texture and colour (Supplementary Figure 1, Supplementary Table 1 and 2). The feeds were provided in separate bowls from 2 days of age onwards. Feed A was an 8-mm diameter pellet mixed by Research Diet Services (Wijk bij Duurstede, The Netherlands) and extruded using a co-rotating double screw extruder (M.P.F. 50, Baker Perkins, Peterborough, United Kingdom). Extruder settings intendedly varied during production, resulting in differences in pellet texture, length (8-22 $\mathrm{mm})$ and hardness $(7.3-17.7 \mathrm{~kg})$ to create diversity within feed A. Feed B was a 14-mm diameter pellet, with a length of $10-20 \mathrm{~mm}$ and a hardness of $6.8 \mathrm{~kg}$. Feed B could not pass the slats in intact form in comparison to feed A. Pellet hardness was measured with a Kahl pellet hardness tester (Amandus Kahl Nachf, Reinbek, Germany) according to Thomas and Van der Poel (1996) using 10 pellets for feed B and 10 pellets per production setting for feed 
A.

The other group of piglets (flavour novelty (FN), $n=9$ litters) received feed $A$ only in both bowls from 2 days of age. From day 6 of age flavours (i.e. substances to influence the sensory perception of the feed as related to its taste and smell) were added to feed $A$ in one bowl in a daily sequential order. The flavours were mixed through the feed at a predetermined rate according to the manufacturer's advice and small human flavour tests. The flavours were anise $(0.08 \mathrm{~g} / \mathrm{kg})$, vanilla $(0.35 \mathrm{~g} / \mathrm{kg})$, red fruit $(0.5 \mathrm{~g} / \mathrm{kg})$ and an essential oil mixture (EOM, $0.4 \mathrm{~g} / \mathrm{kg}$ ) (Provimi, Cargill Animal Nutrition, Rotterdam, The Netherlands). The main components of the EOM are essential oil compounds from cinnamon, clove and oregano. Over the lactation period, each flavour was fed four times, once in each of four 4day blocks.

Litters were allotted to one of two treatment groups by sow's parity (DD: $3.6 \pm 0.5$, range: 2 to $7 ; \mathrm{FN}: 3.4 \pm 0.6$, range: 1 to 7 ) and average weight of the litter at day 1 of age (DD: $1.3 \pm 0.06 \mathrm{~kg} /$ piglet; FN: $1.4 \pm 0.06 \mathrm{~kg} /$ piglet) and treatment groups were randomly distributed within farrowing rooms. DD-sows had litters of $14.0 \pm 0$ piglets and FN-sows had litters of $14.0 \pm 0.17$ piglets (range: 13 to 15 ) at the start of dietary treatments. One piglet died after allocation to the treatments. Weaning age did not differ between treatment groups (DD: $22.5 \pm 0.06$ days of age; FN: $22.2 \pm 0.09$ days of age).

\subsection{Measurements}

\subsubsection{Piglet performance}

Piglets were individually weighed at 2 days of age (before commencing creep feeding), at 6 days of age (before commencing flavour novelty in $\mathrm{FN}$ ) and at 22 days of age (at weaning). Creep feed intake was determined per pen per feed type (in grams) at day 6, 12, 16 and 22 for DD-litters and daily from day 6 onwards for FN-litters. This was done by weighing feed remains in the feed bowl and on the floor. The intake per feed type was also calculated as a percentage of the total feed intake to determine the proportional intake of the feed types. 
A subset of litters ( $n=11$, from one room) was used to study feed-related behaviours. Piglets were marked (from 1 to 14 per litter) the day before observations using dark permanent hair dye. Live behavioural observations were done at 9, 14 and 21 days of age using 4-min instantaneous scan sampling for 6 sessions of one hour per day (i.e. 90 scans per piglet per day). Observations were performed in the morning from 8:15 to 9:15h, 9:30 to $10: 30 \mathrm{~h}, 10: 45$ to $11: 45 \mathrm{~h}$ and in the afternoon from $13: 45$ to $14: 45 \mathrm{~h}, 15: 00$ to $16: 00 \mathrm{~h}$ and $16: 15$ to $17: 15 \mathrm{~h}$. Feeding behaviours were scored by two observers using a Psion hand-held computer with the Pocket Observer 3.1 software package (Noldus Information Technology, Wageningen, The Netherlands). The ethogram is given in Table 1. Observations were also used to discriminate 'eaters', i.e. piglets scored eating creep feed (from the bowl and/or floor) at least once, from 'non-eaters' per observation day. The percentage of eaters was calculated by dividing the number of eaters per litter by the total amount of piglets in the same litter at that observation day. In addition, eaters were grouped into different eater classes (i.e. good, moderate and bad) after Collins et al. (2013). Piglets that were observed eating on all 3 observation days (day 9, 14 and 21 of age) were classified as 'good eaters'. 'Moderate eaters' were observed eating on 2 out of 3 observation days and 'bad eaters' were observed eating only 1 out of 3 observation days. Piglets that were never seen eating were classed as 'non-eaters'. If a piglet was scored as eater, it was also investigated which feed types it consumed throughout lactation.

\subsection{Statistical analyses}

Data were analysed with the statistical software SAS 9.3 (SAS Institute Inc., Cary, NC, USA). Behavioural variables were expressed as proportions of time. Exploring feed (bowl), exploring feed on the floor and playing with feed were pooled into 'exploring creep feed'. Eating and eating feed from the floor were merged into 'eating creep feed'. The behaviours exploring sow feed and eating sow feed were combined into 'interest in sow feed'. Exploring sow trough was excluded from analyses as this behaviour might indicate exploration towards 
the environment and not exploration towards sow feed per se. To investigate 'interest in water', behaviours drinking and exploring drinking nipple were combined. Model residuals were checked for normal distribution. Feed intake data were square root transformed and behavioural data were arcsine square root transformed if needed to meet the assumption of normality. Correlations between feed intake, time spent eating and time spent exploring the feed were calculated at litter level using Spearman's correlation coefficients (PROC CORR). Performance and behavioural data were analysed using repeated-measure mixed models (PROC MIXED). Differences at $P<0.05$ were considered statistically significant and differences at $0.05 \leq P<0.10$ were considered as trend.

Effects of dietary treatment. Models for behaviour included the fixed effects of dietary treatment (DD vs. FN), day and their interaction, with piglet (nested within pen and dietary treatment) as experimental unit and pen (nested within dietary treatment) as random effect. Pen was the experimental unit for analyses of feed intake and the percentage of eaters. To study the effect of dietary treatment on body weight gain (day 2-22) during the suckling period dietary treatment was used as fixed effect and pen (nested within dietary treatment) as random effect. Moreover, a Fisher's Exact Test (PROC FREQ) was performed to test whether eater classification was affected by dietary treatment.

Effects of feed type within dietary treatment. To study effects on behaviour and feed intake within dietary treatment, feed type (A vs. B in DD and A vs. A + flavours in FN), day and their interaction were used as fixed effects. Furthermore, to test flavour preferences within FN, the daily intake of each of the four flavoured feeds per pen (after correcting for the total feed intake on that day) was analysed including flavour (anise, vanilla, red fruit, EOM), 4-day block (day 6-10, 10-14, 14-18, 18-22 of age) and their interaction as fixed effects. Significant fixed effects were further analysed using differences of least squares means, with Tukey adjustment for multiple comparisons. Feed intake data were also analysed per period (2-6, 6-12, 12-16 and 16-22 days of age) and over the whole suckling period (2-22 days of age) using mixed models with dietary treatment or feed type as fixed 
effect. (Untransformed) data are presented as means \pm SEM (based on pen averages for body weight (gain) and behavioural variables).

\section{Results}

\subsection{Piglet performance}

Irrespective of dietary treatment, feed intake $(P<0.0001)$ increased with age. Feed intake highly correlated with time spent eating at litter level $(r=0.91 ; P<0.0001)$, but there was no correlation between feed intake and time spent exploring the feed $(r=-0.14 ; P=0.45)$.

Effects of dietary treatment. DD-litters (14.0 \pm 0 piglets) ate more than FN-litters (14.0 \pm 0.17 piglets) in the two weeks before weaning (Figure 1). Total feed intake during lactation also differed between DD and FN-litters (DD: $3.0 \pm 0.32 \mathrm{~kg}$ vs. FN: $2.0 \pm 0.12 \mathrm{~kg} / \mathrm{litter} ; P=$ 0.02) and varied between individual litters (DD: range 1.9-4.7 kg; FN: 1.6-2.7 kg/litter).

Dietary treatment, however, did not affect body weight gain from d2-22 (DD: $4.7 \pm 0.11 \mathrm{~kg} v$ s. FN: $4.6 \pm 0.17 \mathrm{~kg} /$ piglet; $P=0.71$ ). At weaning, DD-piglets weighed $6.2 \pm 0.13 \mathrm{~kg}$ and FNpiglets weighed $6.2 \pm 0.21 \mathrm{~kg}$.

Also feed $\mathrm{A}$, the common feed used in DD and FN, was eaten more in DD compared to FN (Figure 2). When analysed per period, a significantly higher intake of feed A in DDlitters within d6-12 $(P=0.02)$ and d16-22 $(P=0.04)$ and a numerically higher intake of feed $\mathrm{A}$ in DD-litters within d12-16 $(P=0.17)$ relative to FN-litters was found. Total intake of feed A during lactation did also differ between DD and FN-litters (DD: $1.4 \pm 0.16 \mathrm{~kg}$ vs. FN: $1.0 \pm$ $0.07 \mathrm{~kg} / \mathrm{litter} ; P=0.04)$.

The percentage of piglets that consumed the feed increased over time and did not differ between DD and FN-litters (Figure 3). Once scored as an eater, piglets remained eaters at subsequent observation days, with the exception of five out of 146 piglets. The number of piglets classified as bad eaters was lower for DD (good: 18 (22.2\%), moderate: 46 (56.8\%), bad: 13 (16.0\%) and non-eaters: 4 (4.8\%)) in comparison to FN (good: 13 (18.9\%), 
moderate: 35 (50.7\%), bad: 21 (30.4\%), and non-eaters: $0(0 \%)$; $P<0.05)$. A small number of eaters was observed sampling only one of the two feed types offered (DD: 8 out of 77 eaters vs. FN: 9 out of 69 eaters), whereas the others sampled both feeds.

Effects of feed type within dietary treatment. The proportional intake of feed A and B within DD and feed $A$ with and without additional flavours within $\mathrm{FN}$ was determined during the suckling period to test feed type preferences (Figure 4). Feed type $x$ day $(P=0.29)$ or feed type $(P=0.16)$ did not affect the feed intake of DD-piglets. In accordance, DD-piglets had no preference for feed A $(1.4 \pm 0.16 \mathrm{~kg} / \mathrm{litter})$ or $\mathrm{B}(1.6 \pm 0.18 \mathrm{~kg} / \mathrm{litter} ; P=0.31)$ over the whole suckling period.

FN-piglets preferred feed A with additional flavours over feed A without additional flavours between d16-22 $(P=0.04)$, but not at earlier time points (Feed type $\mathrm{x}$ day: $P=0.02$; feed type: $P=0.104)$. Specifically, this preference occurred the day that feed $A$ was supplemented with red fruit (i.e. 20 days of age) in the 4-day block from day 18 to 22 ( $P<$ 0.0001), as red fruit was clearly eaten more within the flavour novelty treatment compared to the other three flavours in this period (Red fruit: $73 \pm 3.3 \%$; anise: $54 \pm 4.4 \%$; vanilla: $47 \pm 3.4$ \%; EOM: $41 \pm 4.6 \%$ of total intake/pen/day, flavour $\times 4$-day block: $P=0.01$ ). No overall preference for feed A with additional flavours $(1.1 \pm 0.06 \mathrm{~kg} / \mathrm{litter})$ was found in FN compared to feed A without additional flavours $(0.9 \pm 0.07 \mathrm{~kg} / \mathrm{litter}, P=0.14)$.

\subsection{Behaviours}

Effects of age. Irrespective of dietary treatment, piglets' behavioural activity was affected by age (Figure 5), except for exploring the feed $(P=0.18)$.

Time spent on 'suckling behaviour' decreased with time. Nine-day-old piglets spent more time massaging the udder than 14-day-old $(P=0.05)$ and 21-day-old piglets $(P=$ 0.003; d9: $14.8 \pm 1.15 \%$ of observations; d14: $13.6 \pm 1.10 \%$; d21: $13.2 \pm 0.92 \%)$. In addition, suckling significantly differed between day 9,14 and 21 (d9: $4.3 \pm 0.72 \%$ of 
observations; d14: $2.2 \pm 0.51 \%$; d21: $1.7 \pm 0.43 \% ; P<0.01$ for all). On the other hand, time spent on 'ingestive behaviour' increased in time. Time spent eating creep feed increased from day 9 to day 14 and 21 (d9: $0.4 \pm 0.17 \%$ of observations; d14: $1.7 \pm 0.23 \%$; d21: $6.0 \pm$ $0.68 \% ; P<0.0001$ for all). Moreover, nine-day-old piglets had less interest in sow feed compared to 14-day-old $(P<0.0001)$ and 21-day-old piglets $(P<0.0001 ;$ d9: $0.13 \pm 0.06 \%$ of observations; d14: $0.66 \pm 0.17 \%$; d21: $0.48 \pm 0.11 \%)$. Interest in water tended to be less for nine-day-old piglets relative to 14-day-old piglets $(P=0.052)$ and was significantly different between the other time points ( $d 9: 0.38 \pm 0.10 \%$ of observations; $d 14: 0.67 \pm 0.12$ $\% ;$ d21: $0.92 \pm 0.09 \% ; P<0.01)$.

Effects of dietary treatment. A dietary treatment $x$ day interaction was found for suckling $(P=0.0003)$. Although no differences were observed using least squares means, suckling behaviour was numerically higher for DD-piglets at 9 days of age, but numerically lower at 14 days of age compared to FN-piglets respectively. DD-piglets spent more time exploring the feed compared to FN-piglets at 14 days of age (DD: $1.54 \pm 0.21 \%$ vs. FN: 0.55 $\pm 0.08 \% ; P=0.001$ ), but no differences were found in time spent eating the feed. In addition, no effects of dietary treatment were found during the suckling period on time spent massaging the udder of the sow, interest in water and interest in sow feed $(P>0.10)$.

Effects of feed type within dietary treatment. DD-piglets explored feed B more compared to feed A (Feed B: $0.83 \pm 0.04 \%$ vs. Feed A: $0.43 \pm 0.07 \%$; feed type: $P=0.001$ ). Within DD-litters, no feed type $x$ day interactions were found for exploring the feed $(P=0.66)$ or eating $(P=0.61)$ and no effect of feed type was found on eating $(P=0.78)$.

The feed type $x$ day interaction affected the eating behaviour of FN-piglets $(\mathrm{P}<$ 0.0001), but not their exploratory behaviour towards the feed $(P=0.17)$. Within FN-litters, piglets tended to be more frequently observed eating feed $\mathrm{A}$ without additional flavour compared to feed $A$ with additional flavour (i.e. EOM) at 21 days of age (A: $3.80 \pm 0.74 \%$ vs. A + flavours: $1.92 \pm 0.39 \% ; P=0.054)$, which corresponds to the feed intake measures on that day (EOM: $41 \pm 4.6 \%$ of total intake/pen at day 21). No effects of feed type were found 
on eating $(P=0.12)$ and exploring the feed $(P=0.77)$ within $\mathrm{FN}$-litters during the suckling period.

\section{Discussion}

In this study we compared the effects of dietary diversity and flavour novelty on the feeding behaviour and performance of suckling piglets. Provision of feed $A$ and $B$ increased preweaning feed intake by $50 \%$ compared to provision of feed A only (with and without additional flavours). Yet, piglets receiving feed $A$ and $B$ had no overall preference in terms of feed intake for either feed A or B, indicating pre-weaning feed intake increased by an enhanced intake of both feeds. These results support our hypothesis that the more diverse the feeds provided in terms of sensory properties (e.g. ingredient composition, texture), the greater the intake will be. The reason for this is expected to be sensory-specific satiety and/or piglets' intrinsic motivation to explore. Alternatively, differences in nutrient profiles between the two treatments may have exerted physiological effects that may have influenced feed ingestion.

Sensory-specific satiety involves a rapid and significant decline in pleasantness of taste, smell, appearance and texture of eaten feed in comparison to the pleasantness of noneaten feed, as reviewed by Rolls (1986). To maintain feed intake at a high level, the feeds provided should therefore vary along as many properties as possible, emphasizing on contrasts, to reduce sensory-specific satieties that impair palatability. Most of the eaters were observed consuming both of the feed types of choice. Therefore it appears that in a choicecondition, piglets prefer a varied diet rather than sampling from just one feed (feed A or B; feed A with or without additional flavours).

Although behavioural observations did not show a significant difference between DDand FN-piglets in terms of time spent eating creep feed, the exploratory behaviour towards the creep feed was higher for DD-piglets compared to FN-piglets at 14 days of age. Several studies have suggested that feed exploration is beneficial for feed intake in the pre-weaning period 
347 (Adeleye et al., 2014; Kuller et al., 2010; Van den Brand et al., 2014). It should be noted, 348 though, that feed intake was not significantly correlated with feed exploration on the same day 349 (at litter level) in this study. In addition, no day effect was found for exploring creep feed, suggesting exploratory foraging behaviour remains important for piglets throughout lactation. Within DD-litters, piglets explored feed B more compared to feed A, which may suggest that increased exploration of one feed, stimulated feed intake of both feeds. Feed B is a large diameter pellet which has been suggested to be easier to pick up, hold or carry in the mouth of young piglets compared to smaller diameter pellets (Van den Brand et al., 2014).

One could hypothesize that feed exploration encourages the development of feed handling skills which are needed for ingestion and thereby increased exploration may increase the percentage of eaters. The percentage of eaters, however, did not differ between DD and FN and the higher feed intake for DD-litters can therefore be explained by a higher intake per piglet, supported by less bad eaters in the DD compared to FN group. One should notice that the percentage of eaters was remarkably high in this study compared to previous studies (e.g. Collins et al., 2013 (d16: 41\%, d19: 50\%, d21: 77\%); Pluske et al., 2007 (d19: 49\%, d23: 72\%); Sulabo et al., 2010 (d14: 20\%, d21: 57\%); Tucker et al., 2010 (d10: 1.4\%, d14: 4.6\%, d21: $29 \%)$ ), which may have been caused by applying diversity and novelty to the piglets' diet. Nevertheless, this remains to be shown in comparison to a control group (no-variety condition), which was absent in this study. Another possible explanation might be a difference in method, as most studies used a colour marker in the feed, such as chromic oxide, to detect consumption of creep feed. One may not be able to detect the colour marker in the faeces of piglets with a very low creep feed intake as the large amount of sow's milk may mask the colour (Barnett et al., 1989) or of piglets with an irregular feed intake pattern (Kuller et al., 2007). Two other studies have determined the percentage of eaters using behavioural observations, but used a lower number and distribution of scans per observation day (Delumeau and MeunierSalaün, 1995 (5-min scan sampling, 90 min/d); Devillers and Farmer, 2009 (1-min scan sampling, $60 \mathrm{~min} / \mathrm{d})$ ), which may have led to false-negative results. On the other hand, these 
studies used a broader definition of eating in comparison to this study, which may have led to false-positive results.

DD-piglets had a higher feed intake compared to FN-piglets, but weight gain before weaning was not affected. A possible explanation for this result is that pre-weaning weight gain is mainly determined by piglets' milk intake during lactation (Adeleye et al., 2014) and time spent suckling did not differ between DD- and FN-piglets. The relatively short duration of feed provision (i.e. weaning at three weeks of age) would be another possible explanation. Creep feed intake is known to follow an exponential pattern (Pluske et al., 2007). Therefore, a greater pre-weaning feed intake may increase body weight gain only shortly before weaning at a later age (Bruininx et al., 2004; Pluske et al., 2007). Besides, the purpose of a high feed intake before weaning is mainly to facilitate body weight gain after weaning due to its' expected benefits for post-weaning feed intake. Even a small improvement in total creep feed intake per piglet (64 g/piglet) has been shown to be advantageous for post-weaning growth $(1 \mathrm{~kg} / \mathrm{piglet}$ in 2 weeks post-weaning; Adeleye et al., 2014).

In the study of Adeleye et al. (2014), litters that were fed creep feed to which different flavours were added in a daily sequential order had a higher hourly frequency of feeder visits and a doubled feed intake compared to control litters which received the same creep feed without additional flavours. By simultaneously providing feed with and without additional successive flavours within a litter by the use of a choice test, we found that feed exploration and intake in general did not differ between feed with or without additional flavours. FN-piglets consumed more of feed $A$ with additional flavours relative to feed $A$ without additional flavours in the last six days before weaning however, which seemed driven by the three times higher intake of red fruit that was observed at day 20 . The reason why the piglets chose to consume more of red fruit flavoured feed only at 20 days of age could not be clearly explained. Although it is difficult to compare flavour preferences between studies with a different experimental setup, red fruit was most preferred compared to vanilla, anise and EOM in this study, but it was least preferred compared to butterscotch, apricot, toffee and apple in Adeleye et al. (2014). 

without additional flavours) as well as successive variety (novel flavours added over time) in comparison to DD (i.e. the dietary diversity treatment) which only involved simultaneous variety (feed $A$ and feed B). On one hand, the successive exposure to novel flavours on a daily basis likely involved an initial fear response and reluctance by the piglets to try the novel flavoured feed (Oostindjer et al., 2011) before overcoming neophobia and ingesting the feed. On the other hand, piglets are highly curious animals and were found to seek out for novelty if provided the choice between novelty or familiarity (Wood-Gush and Vestergaard, 1991). No clear evidence for (attenuation of) neophobia toward the novel flavours was found in this study, as the proportional intake of feed A with flavours generally did not increase in time. Dietary diversity seems therefore a more likely cause for the feed intake differences among the treatments, but a possible effect of food neophobia on feed intake cannot be fully excluded. The balance between aversion and acceptance of flavoured creep feed in piglets seems complex, as feed intake of either a familiar or unfamiliar flavour is variable (e.g. Blavi et al., 2016; Figueroa et al., 2013; Langendijk et al., 2007). These inconsistent results indicate that it is hard to predict whether a flavour increases acceptance of the feed or results in aversion and reduced feed intake.

\section{Conclusion}

In conclusion, this study showed that provision of dietary diversity to suckling piglets stimulated their feed exploration and intake more than dietary flavour novelty, but did not enhance the percentage of piglets within a litter that consume the feed (at an early age) or their growth performance during the pre-weaning period. Future research will investigate the effect of dietary diversity on the (feeding) behaviour and performance of suckling piglets versus a control group (no-variety condition) and will study the adaptive capacity of these piglets in novelty tests and during the post-weaning period. The amount of solid feed consumed during the suckling period has been shown to correlate positively with the amount of solid feed 
consumed during the initial weaning period, as well as with the growth performance of newly weaned pigs (Berkeveld et al., 2007; Kuller et al., 2004). Moreover, dietary variety in early life enhanced the acceptance rate of novel feeds and novel flavours (Catanese et al., 2012; Villalba et al., 2012) and reduced the fear response to a novel environment (Villalba et al., 2012), as shown in weaned lambs. It is therefore hypothesized that early exposure to dietary variety can increase adaptability in novel situations. Inclusion of dietary variety in piglet rearing during lactation may therefore be of particular interest at weaning at which rapid acceptance and high intake of novel feed in a new environment is needed to prevent gastro-intestinal dysfunction and associated health problems and production losses.

\section{Acknowledgments}

This study is part of the research programme 'Genetics, nutrition and health of agricultural animals' with project number 868.15 .010 , which is financed by the Netherlands Organisation for Scientific Research, Cargill Animal Nutrition (CAN) and Coppens Diervoeding. The authors thank Fleur Bartels and personnel of CAN Innovation Center in Velddriel for their help with the experiment. We like to acknowledge CAN for the use of their research facilities and for providing flavouring agents and Coppens Diervoeding for providing feed $\mathrm{B}$. The authors are grateful to Tamme Zandstra for producing feed A and pellet hardness measurements and to Evelien Alderliesten, Moniek van den Bosch and Henry van den Brand for their advice. 


\section{References}

450

451

452

453

454

455

456

457

458

459

460

461

462

463

464

465

466

467

468

469

470

471

472

473

Adeleye OO, Guy JH and Edwards, SA 2014. Exploratory behaviour and performance of piglets fed novel flavoured creep in two housing systems. Animal Feed Science and Technology 191, 91-97.

Barnett KL, Kornegay ET, Risley CR, Lindemann MD and Schurig GG 1989.

Characterization of creep feed consumption and its subsequent effects on immune response, scouring index and performance of weanling pigs. Journal of Animal Science $67,2698-2708$.

Berkeveld M, Langendijk P, Van Beers-Schreurs HMG, Koets AP, Taverne MAM and Verheijden JHM. 2007. Postweaning growth check in pigs is markedly reduced by intermittent suckling and extended lactation. Journal of Animal Science 85, 258-266.

Blavi L, Solà-Oriol D, Mallo JJ and Pérez JF 2016. Anethol, cinnamaldehyde, and eugenol inclusion in feed affects postweaning performance and feeding behavior of piglets. Journal of Animal Science 94, 5262-5271.

Bruininx EM, Schellingerhout AB, Binnendijk GP, Van der Peet-Schwering CMC, Schrama JW, den Hartog LA, Everts H and Beynen AC 2004. Individually assessed creep feed consumption by suckled piglets: influence on post-weaning food intake characteristics and indicators of gut structure and hind-gut fermentation. Animal Science 78, 67-75.

Bruininx EM, Binnendijk GP, Van der Peet-Schwering CMC, Schrama JW, den Hartog LA, Everts $\mathrm{H}$ and Beynen AC 2002. Effect of creep feed consumption on individual feed intake characteristics and performance of group-housed weanling pigs. Journal of Animal Science 80, 1413-1418.

Carstensen L, Ersbøll AK, Jensen KH and Nielsen JP 2005. Escherichia coli post-weaning diarrhoea occurrence in piglets with monitored exposure to creep feed. Veterinary Microbiology 110, 113-123. 
Catanese F, Distel RA, Provenza FD and Villalba JJ 2012. Early experience with diverse foods increases intake of nonfamiliar flavors and feeds in sheep. Journal of Animal Science 90, 2763-2773.

Centraal Veevoeder Bureau (Dutch Central Bureau for Livestock Feeding, CVB) 2007. CVB Table Pigs 2007. Chemical composition and nutritional value of feedstuffs and feeding standards. CVB series no 36, Product Board Animal Feed, The Hague, The Netherlands.

Collins CL, Morrison RS, Smits RJ, Henman DJ and Dunshea FR 2013. Interactions between piglet weaning age and dietary creep feed composition on lifetime growth performance. Animal Production Science 53, 1025-1032.

Delumeau O and Meunier-Salaün MC 1995. Effect of early trough familiarity on the creep feeding behaviour in suckling piglets and after weaning. Behavioural Processes 34, 185-196.

Devillers N and Farmer C 2009. Behaviour of piglets weaned at three or six weeks of age. Acta Agriculturae Scandinavica Section A. Animal Science 59, 59-65.

Distel RA, Iglesias RMR, Arroquy J and Merino J 2007. Note on increased intake in lambs through diversity in food flavor. Applied Animal Behaviour Science 105, 232-237.

Figueroa J, Solà-Oriol D, Vinokurovas L, Manteca X and Pérez JF 2013. Prenatal flavour exposure through maternal diets influences flavour preference in piglets before and after weaning. Animal Feed Science and Technology 183, 160-167.

Gundlach H 1968. Brutftirsorge, Brutplege, Verhaltensontogenese und Tagesperiodik beim Europiischen Wildschwein (Sus scrofa L. ). Zeitschrift für Tierpsychologie 25, 955995.

Hanson RP and Karstad L 1959. Feral swine in the southeastern United States. Journal of Wildlife Management 23, 64-74.

Heo JM, Opapeju FO, Pluske JR, Kim JC, Hampson DJ and Nyachoti CM 2013. Gastrointestinal health and function in weaned pigs: a review of feeding strategies to 
control post-weaning diarrhoea without using in-feed antimicrobial compounds. Journal of Animal Physiology and Animal Nutrition 97, 207-237.

Klindt J 2003. Influence of litter size and creep feeding on preweaning gain and influence of preweaning growth on growth to slaughter in barrows. Journal of Animal Science 81, 2434-2439.

Kuller WI, Soede NM, Van Beers-Schreurs HMG, Langendijk P, Taverne MAM, Kemp B and Verheijden JHM 2007. Effects of intermittent suckling and creep feed intake on pig performance from birth to slaughter. Journal of Animal Science 85, 1295-1301.

Kuller WI, Soede NM, Van Beers-Schreurs HMG, Langendijk P, Taverne MAM, Verheijden JHM and Kemp B 2004. Intermittent suckling: Effects on piglet and sow performance before and after weaning. Journal of Animal Science 82, 405-413.

Kuller WI, Tobias TJ and Van Nes A 2010. Creep feed intake in unweaned piglets is increased by exploration stimulating feeder. Livestock Science 129, 228-231.

Langendijk P, Bolhuis JE and Laurenssen BFA 2007. Effects of pre- and postnatal exposure to garlic and aniseed flavour on pre- and postweaning feed intake in pigs. Livestock Production Science 108, 284-287.

Meynhardt H 1980. Schwarzwild report. Vier Jahre unter Wildschweinen. (In German.). Verlag J. Neumann-Neudamm, Leipzig, Germany.

Oostindjer M, Mas-Muñoz J, Van den Brand H, Kemp B and Bolhuis JE 2011. Maternal presence and environmental enrichment affect food neophobia of piglets. Biology Letters 7, 19-22.

Pajor EA, Fraser D and Kramer DL 1991. Consumption of solid food by suckling piglets: Individual variation and relation to weight gain. Applied Animal Behaviour Science 32, 139-155.

Petersen V 1994. The development of feeding and investigatory behaviour in free-ranging domestic pigs during their first 18 weeks of life. Applied Animal Behaviour Science 42, 87-98. 
Pinna W, Nieddu G, Moniello G and Cappai MG 2007. Vegetable and animal food sorts found in the gastric content of Sardinian Wild Boar (Sus scrofa meridionalis). Journal of Animal Physiology and Animal Nutrition 91, 252-255.

Pluske JR, Kim JC, Hansen CF, Mullan BP, Payne HG, Hampson DJ, Callesen J and Wilson $\mathrm{RH}$ 2007. Piglet growth before and after weaning in relation to a qualitative estimate of solid (creep) feed intake during lactation: A pilot study. Archives of Animal Nutrition 61, 469-480.

Raynor HA and Epstein LH 2001. Dietary variety, energy regulation and obesity. Psychological Bulletin 127, 325-341.

Rolls BJ 1986. Sensory-specific satiety. Nutrition Reviews 44, 93-101.

Rolls BJ, Rowe EA, Rolls ET, Kingston B, Megson A and Gunary R 1981. Variety in a meal enhances food intake in man. Physiology \& Behavior 26, 215-221.

Rolls BJ, Van Duijvenvoorde PM and Rowe EA 1983. Variety in the diet enhances intake in a meal and contributes to the development of obesity in the rat. Physiology \& Behavior $31,21-27$.

Sander SJ, Bullermann J, Arlinghaus M, Verspohl J and Kamphues J 2012. The influence of grinding intensity and compaction of diets on the microbial community in the gastrointestinal tract of young pigs. Journal of Animal Science 90, 16-18.

Sulabo RC, Jacela JY, Tokach MD, Dritz SS, Goodband RD, DeRouchey JM and Nelssen JL 2010. Effects of lactation feed intake and creep feeding on sow and piglet performance. Journal of Animal Science 88, 3145-3153.

Thomas M and Van der Poel AFB 1996. Physical quality of pelleted animal feed. 1. Criteria for pellet quality. Animal Feed Science Technology 61, 89-112.

Torrallardona D, Andrés-Elias N, López-Soria S, Badiola I and Cerdà-Cuéllar M 2012. Effect of feeding different cereal-based diets on the performance and gut health of weaned piglets with or without previous access to creep feed during lactation. Journal of Animal Science 90, 31-33. 
555 Treit D, Spetch ML and Deutsch JA 1983. Variety in the flavor of food enhances eating in the rat: controlled demonstration. Physiology \& Behavior 30, 207-211.

Tucker AL, Duncan IJH, Millman ST, Friendship RM and Widowski TM 2010. The effect of dentition on feeding development in piglets and on their growth and behavior after weaning. Journal of Animal Science 88, 2277-2288.

Van den Brand H, Wamsteeker D, Oostindjer M, Van Enckevort LCM, Van der Poel AFB, Kemp B and Bolhuis JE 2014. Effects of pellet diameter during and after lactation on feed intake of piglets pre- and post-weaning. Journal of Animal Science 92, 41454153.

Van der Meulen J, Koopmans SJ, Dekker RA and Hoogendoorn A 2010. Increasing weaning age of piglets from 4 to 7 weeks reduces stress, increases post-weaning feed intake but does not improve intestinal functionality. Animal 4, 1653-1661.

Villalba JJ, Bach A and Ipharraguerre IR 2011. Feeding behavior and performance of lambs are influenced by flavor diversity. Journal of Animal Science 89, 2571-2581.

Villalba JJ, Catanese F, Provenza FD and Distel RA 2012. Relationships between early experience to dietary diversity, acceptance of novel flavors, and open field behavior in sheep. Physiology \& Behaviour 105, 181-187.

Wood-Gush DGM and Vestergaard KS 1991. The seeking of novelty and its relation to play. Animal Behaviour 42, 599-606. 
Table 1. Feeding behaviours of piglets during the suckling period

577 Figure 1. Total feed intake ( $\mathrm{g}$ ) per day of litters offered feed A and B in a choice-test of the 578 dietary diversity treatment (DD, $\mathrm{n}=10$ pens) and litters offered feed $\mathrm{A}$ with and without 579 additional flavours in a choice-test of the flavour novelty treatment ( $F N, n=9$ pens) from commencing creep feeding (at 2 days of age) until weaning (at 22 days of age). One of 4 flavours were added daily to one bowl of feed A from day 6 onwards in FN. Data are means \pm SEM. Asterisks indicate significant $(P<0.05)$ effect of dietary treatment per feed intake period (2-6, 6-12, 12-16 and 16-22 days of age).

Figure 2. Daily intake ( $g$ ) of feed $A$ by litters offered feed $A$ and $B$ in a choice-test of the dietary diversity treatment (DD, n=10 pens) and litters offered feed $A$ with and without additional flavours in a choice-test of the flavour novelty treatment ( $F N, n=9$ pens) from commencing creep feeding (at 2 days of age) until weaning (at 22 days of age). One of 4 flavours were added to one bowl of feed A from day 6 onwards in FN. The intake of feed A between 2-6 days of age within FN was calculated as the average intake from both bowls of feed A. Data are means \pm SEM. Asterisks indicate significant $(P<0.05)$ effect of dietary treatment per feed intake period (2-6, 6-12, 12-16 and 16-22 days of age).

Figure 3. Percentage of piglets that consume creep feed at 9, 14 and 21 days of age in litters offered feed $A$ and $B$ in a choice-test of the dietary diversity treatment ( $D D, n=10$ pens) and litters offered feed $A$ with and without additional flavours in a choice-test of the flavour novelty treatment ( $F N, n=9$ pens) from commencing creep feeding (at 2 days of age) until weaning (at 22 days of age). One of 4 flavours were added to one bowl of feed A from day 6 onwards in FN. Data are means \pm SEM. 
601 Figure 4. Ratio between feed A and B within a choice-test of the dietary diversity treatment 602 (DD, $n=10$ litters, panel $A$ ) and between feed $A$ with and without additional flavours within a 603 choice-test of the flavour novelty treatment ( $F N, n=9$ litters, panel B). One of 4 flavours were 604 added to one bowl of feed A from day 6 onwards in FN. Data are means \pm SEM.

605

606 Figure 5. Feed-related behavioural activities (\% of total observations) of suckling piglets 607 offered feed $A$ and $B$ in a choice-test of the dietary diversity treatment $(D D, n=83$ piglets from 6086 litters) and piglets offered feed A with and without additional flavours in a choice-test of the 609 flavour novelty treatment ( $F N, n=69$ piglets from 5 litters) from commencing creep feeding (at 6102 days of age) until weaning (at 22 days of age). One of 4 flavours were added to one bowl 611 of feed A from day 6 onwards in FN. Data are means \pm SEM. Asterisks indicate significant $(P$ $612<0.05)$ effect of dietary treatment per day $(9,14$ and 21 days of age). 

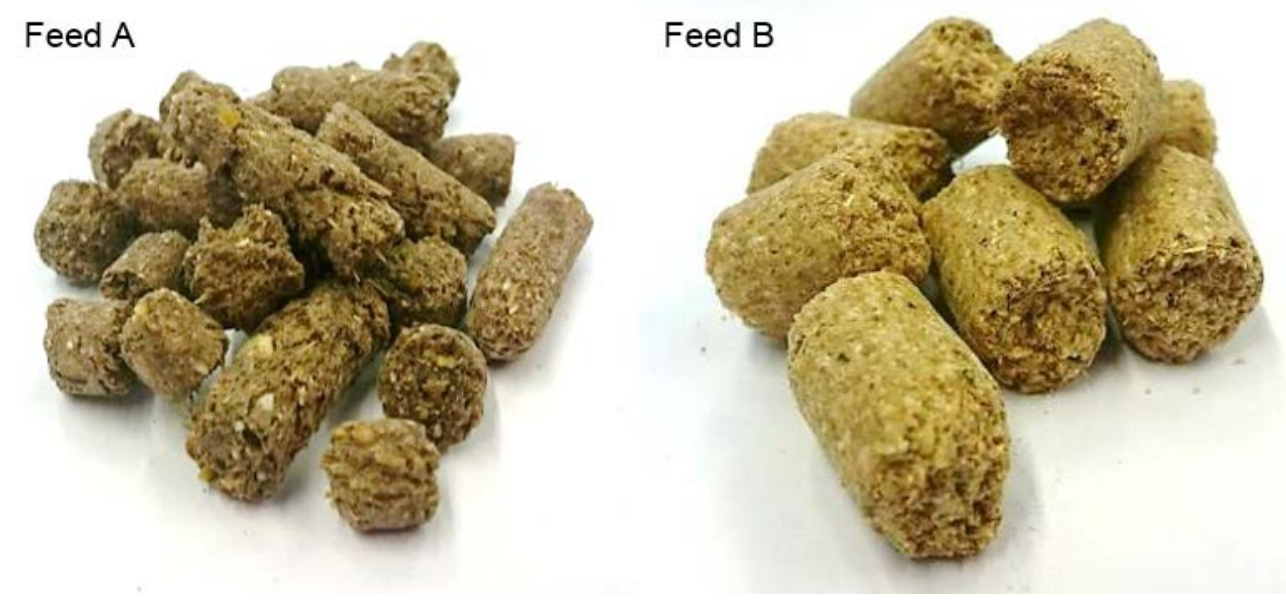

Supplementary Figure 1. Two feeds provided in separate bowls to suckling piglets, receiving either feed $A+B$ in a choice test or feed $A$ only (with and without additional flavours in a choice test). Feed A (Animal Nutrition Group, Wageningen University \& Research, Wageningen, The Netherlands) and B (Baby Big XL, Coppens Diervoeding, Helmond, The Netherlands) differed in production method, size, flavour, ingredient composition and nutrient profile, smell, texture and colour. 
Supplementary Table 1. Nutrient profile of feed A and feed B.

\begin{tabular}{|c|c|c|}
\hline Calculated nutrient composition ${ }^{1}$ & Feed A & Feed \\
\hline Dry matter & 891 & 880 \\
\hline Starch & 290 & 366 \\
\hline $\mathrm{NSP}^{2}$ & 261 & 175 \\
\hline Crude protein & 195 & 140 \\
\hline Crude fat & 61 & 96 \\
\hline Crude fibre & 44 & 42 \\
\hline Crude ash & 57 & 33 \\
\hline Calcium & 9.1 & 2.8 \\
\hline Phosphorus & 6.1 & 3.6 \\
\hline Sodium & 2.2 & 3.5 \\
\hline Standardized ileal digestible lysine & 11.9 & 7.8 \\
\hline Standardized ileal digestible methionine & 4.8 & 2.6 \\
\hline Standardized ileal digestible threonine & 7.1 & 5.2 \\
\hline Standardized ileal digestible tryptophan & 2.4 & 1.7 \\
\hline Net energy & 11.8 & 11.4 \\
\hline
\end{tabular}

${ }^{1}$ According to CVB (2007). Nutrients are presented in g/kg dry matter, except for dry matter $(\mathrm{g} / \mathrm{kg})$ and net energy $(\mathrm{MJ} / \mathrm{kg})$.

${ }^{2}$ Calculated as the difference between dry matter and the sum of starch, sugars, crude protein, crude fat and crude ash. 
Supplementary Table 2. Ingredient composition of feed A.

Ingredient component $\quad \%$

Wheat

21.9

Barley 15

Maize 15

Soy protein concentrate $\quad 7$

Soybeans (heat treated) 5

Galacto-oligosaccharides 5

Potato protein 4

Sugarbeet pulp (dehydrated) $\quad 4$

Oat hulls 4

Inulin 4

Pea starch 4

Soybean oil 3

Blood meal (spray dried) 2

Dicalcium phosphate $\quad 1.7$

Sucrose $\quad 1.5$

Calcium carbonate $\quad 1.0$

Sodium chloride $\quad 0.5$

$\begin{array}{ll}\text { Premix }^{1} & 0.5\end{array}$

$\begin{array}{ll}\text { Potassium bicarbonate } & 0.3\end{array}$

L-lysine hydrochloride $\quad 0.3$

DL-methionine $\quad 0.2$

L-threonine $\quad 0.04$

L-tryptophan $\quad 0.04$

$\begin{array}{ll}\text { Total } & 100\end{array}$

${ }^{1}$ Vitamin and mineral premix (per kg of feed): vitamin A: $10000 \mathrm{IU}$, vitamin D3: $2000 \mathrm{IU}$, vitamin E: $40 \mathrm{mg}$, vitamin $\mathrm{K}: 1.5 \mathrm{mg}$, vitamin B1: $1 \mathrm{mg}$, vitamin B2: $4 \mathrm{mg}$, vitamin B6: $1.5 \mathrm{mg}$, vitamin B12: $0.02 \mathrm{mg}$, niacin: $30 \mathrm{mg}$, D-pantothenic acid: $15 \mathrm{mg}$, choline chloride: $150 \mathrm{mg}$, folate: $0.4 \mathrm{mg}$, biotin: $0.05 \mathrm{mg}$, iron: $100 \mathrm{mg}$, copper: $20 \mathrm{mg}$, manganese: $30 \mathrm{mg}$, zinc: 70 mg, iodine: $0.7 \mathrm{mg}$, selenium: $0.25 \mathrm{mg}$, anti-oxidant: $125 \mathrm{mg}$. 


\begin{tabular}{|c|c|}
\hline Behaviour & Description \\
\hline \multicolumn{2}{|c|}{ ‘Feed-related exploratory behaviour' } \\
\hline Exploring feed (bowl) & $\begin{array}{l}\text { Sniffing, touching or rooting creep feed in the bowl or sniffing, } \\
\text { touching, rooting or chewing on feed bowl }\end{array}$ \\
\hline Exploring feed on floor & Sniffing or touching creep feed on the floor \\
\hline Playing with feed & $\begin{array}{l}\text { Rolling creep feed item over floor, walking around the pen with } \\
\text { feed item, shaking head while having feed item in mouth }\end{array}$ \\
\hline Exploring sow feed & Sniffing or touching feed spilled by the sow on the floor \\
\hline Exploring sow trough & Sniffing, touching, rooting or chewing on feed trough of sow \\
\hline \multicolumn{2}{|l|}{ ‘Ingestive behaviour’ } \\
\hline Eating & Eating or chewing creep feed at the feed bowl \\
\hline Eating feed from floor & $\begin{array}{l}\text { Eating or chewing creep feed from the floor (eaten outside the } \\
\text { feed bowl) }\end{array}$ \\
\hline Eating sow feed & Eating or chewing feed spilled by the sow on the floor \\
\hline Drinking & Drinking from drinking nipple \\
\hline Exploring drinking nipple & Sniffing or touching drinking nipple \\
\hline \multicolumn{2}{|l|}{ ‘Suckling behaviour’ } \\
\hline Massaging udder & Massaging udder with head/nose (up-and-down movements) \\
\hline Suckling & Drinking milk from teat of sow (soft suckling noises) \\
\hline
\end{tabular}




\section{Highlights}

- We studied an innovative feeding strategy to increase solid feed intake pre-weaning

- Dietary diversity stimulated feed intake of suckling piglets more than flavour novelty

- The percentage of eaters was not affected, meaning a higher feed intake per piglet

- Our results support that the more diverse the feeds are, the greater their intake

- Intrinsic exploration and sensory-specific satiety may underlie this 


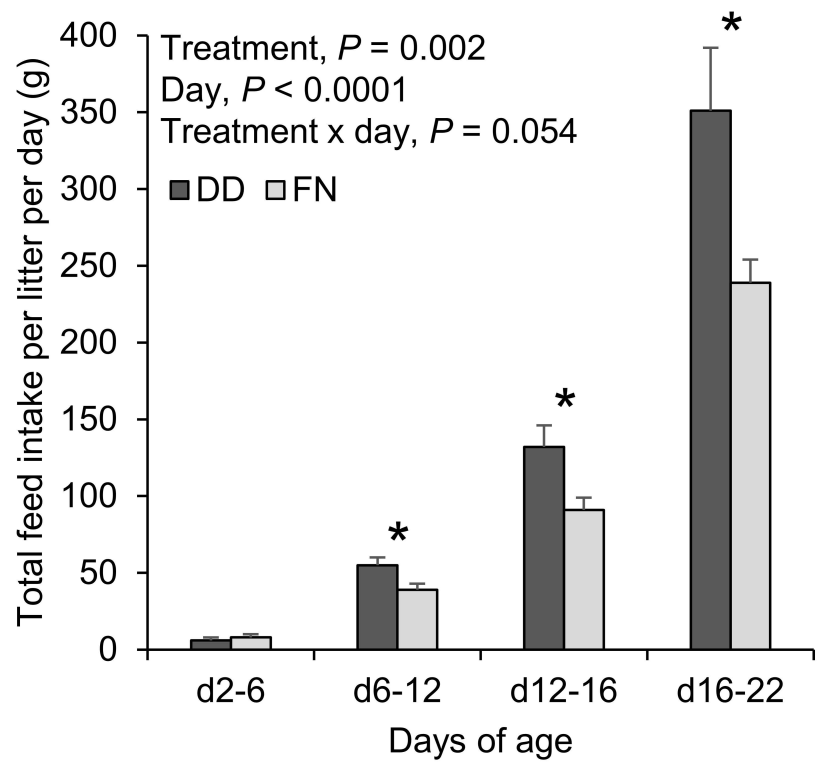




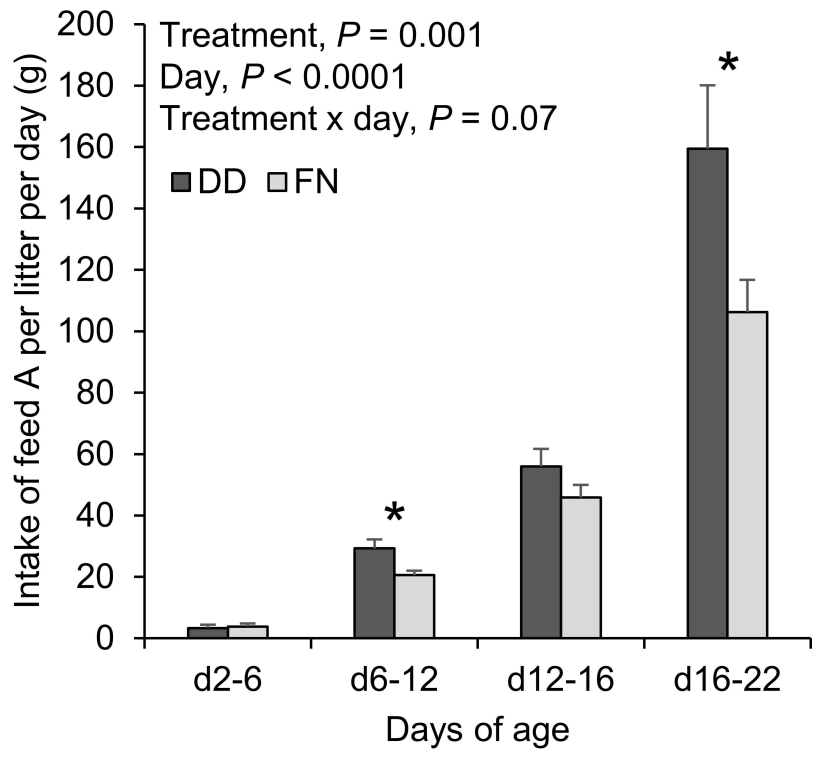




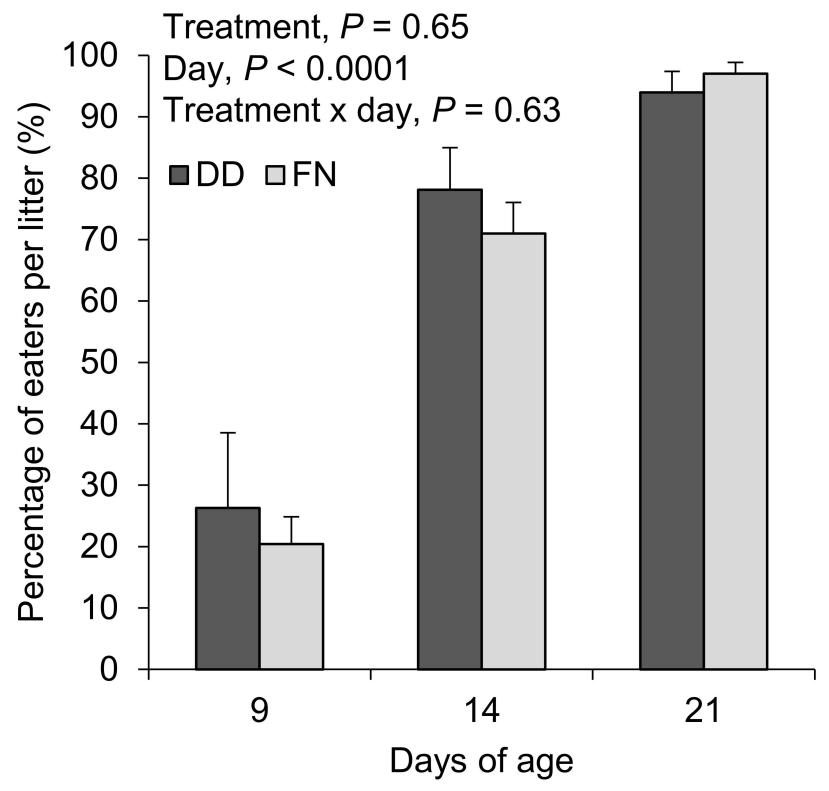


Panel A: DD

Panel B: FN

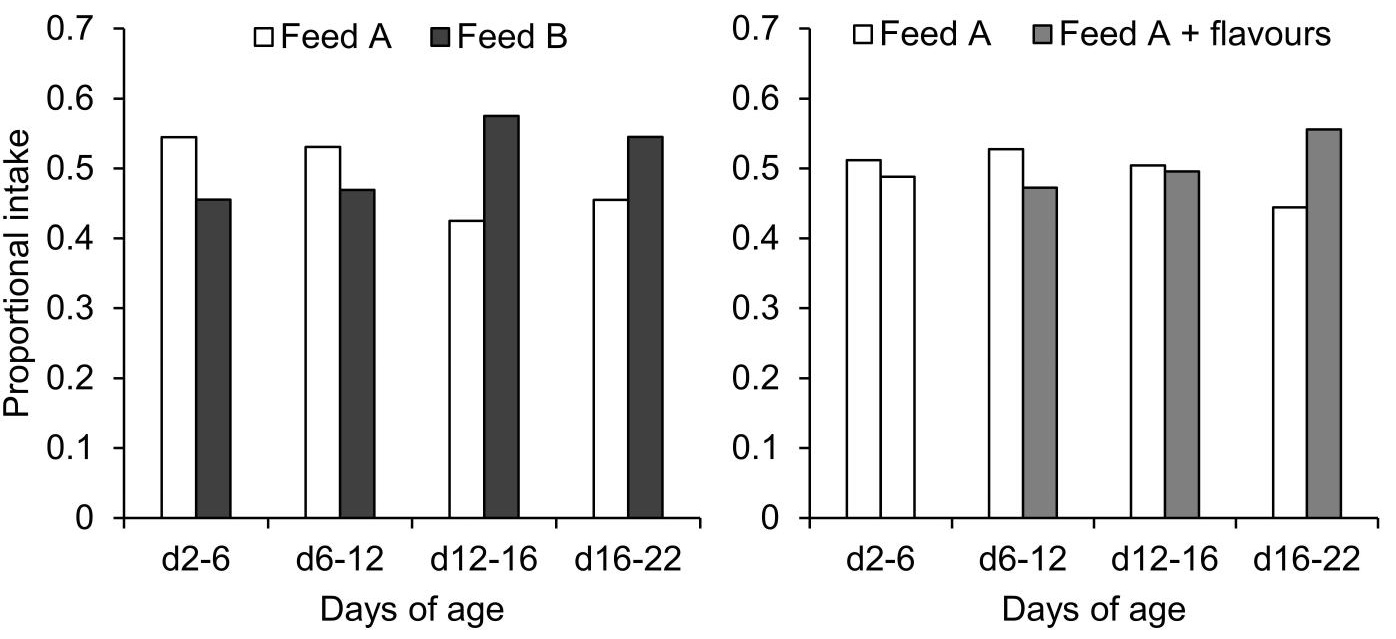



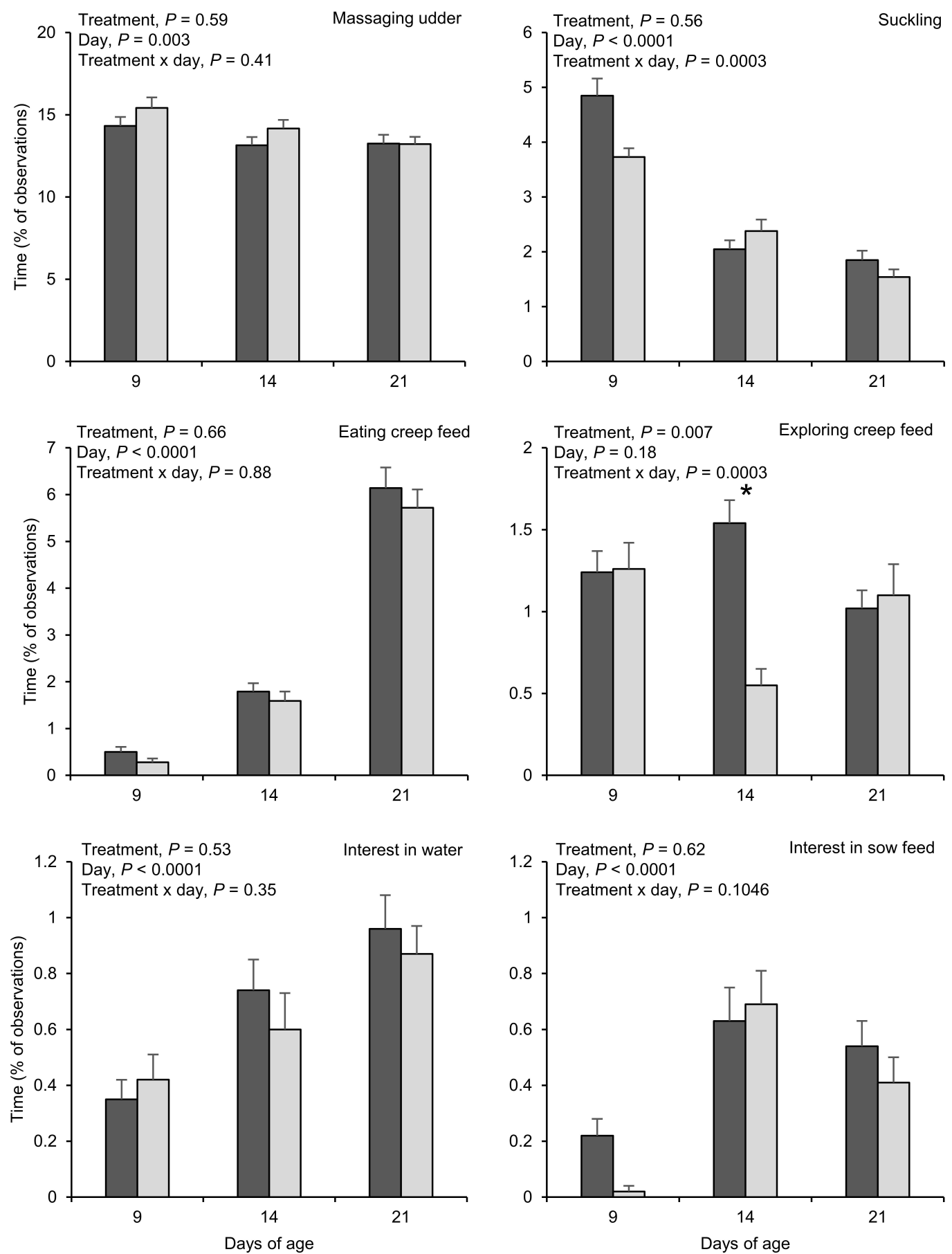